\title{
Unique Expression of the Concept of Time and Space in Wu Qiang New Year Paintings
}

\author{
Jinmin Liu ${ }^{1}$ \\ Shijiazhuang College Arts College \\ Shijiazhuang, Hebei, China, 050035 \\ e-mail: 836119919@qq.com
}

\author{
$\mathrm{Ye} \mathrm{Li}^{2}$ \\ Shijiazhuang College Arts College \\ Shijiazhuang, Hebei, China, 050035
}

\begin{abstract}
As an ancient folk art form, Wu Qiang new year paintings carries people's ideology and unique folk culture in a specific historical period, which also contains a unique expression of the ancestors about time and space. Artists to express their understanding of time and space through the plants, animals, character modeling, character scenes, etc., Studying these unique expressions of time and space in $\mathrm{Wu}$ Qiang new year paintings will provide our modern designs with creative material and stimulate creative inspiration.
\end{abstract}

Keywords-Wu Qiang New Year Paintings; Concept of Time and Space; Plants; Animals; Figures

\section{INTRODUCTION}

As an ancient folk art form, Wu Qiang New Year paintings carry people's ideology and unique folk culture in a specific historical period. Carefully examine the contents of Wu Qiang New Year paintings, you will find they also contain unique expression of the ancestors about time and space. Despite Wu Qiang New Year paintings have a long history, styling and contents will constantly change with flowing of time, the unique understanding of time and space has remained unchanged. In this unique understanding, the integration of the expectations of a happy life and expression of their inner beauty are the expression of their aesthetic appeal.

Art creation of New Year paintings has a unique way of thinking and manifestations, its creation entirely depends on memory rather than painting, the appearance of memories has characteristics of the multi-view, multi-temporal, and the expression of time and space has the arbitrary and highly subjective. After studying the existing Wu Qiang New Year paintings, we found the expressions of the ancestors about time and space are mainly in the following aspects:

\section{DISCUSSED PROBLEMS}

\section{A. Plants are used to express the concept of time and space}

Learning methods are natural, taking methods are natural, which is an important part of life of the original Wuqiang ancestors. They knew the laws of nature, and knew to make

This paper is one of subject results about the art and culture and sciences general project in Hebei Province, "Research on the views of Time and Space of Wu Qiang New Year Paintings", Number: HBWY2014-YG035. use of the laws of nature to benefit, the original ancestors are too familiar with whole nature, and this is a major part of their production, living and entertainment. The ancestors' understanding of time mainly relies on the change of natural laws, cultivate according to the natural climate change, this is their entire life. Which flowers open in what seasons in nature, which has formed the fixed seasonal symbols in their hearts, such as the blooming peony in the spring, lotus in the summer, chrysanthemum in the autumn, plum in the winter, they are typical represents of each season. Artists use flowers to express time and cycle, and use the beauty of the flowers to describe the beauty of life. Express the concept of spacetime with flowers, its performance is the most prominent in window flowers. Such as paper-cut for window decoration in Wuqiang new year paintings, their themes are mainly flowers, the most representative is "Jin Shang Tian Hua", which represents the flowers in four seasons to express their auspicious aspirations in four seasons, here the concept of the four seasons is not only a year, but the expression of the whole time reincarnation, which is that time is eternal, it is forever beautiful like flowers open. "The order and the flowers of the icing on the cake" four words is consistent with the order of opening of flowers, "Jin" is on behalf of peony in the spring, "Shang" represents lotus in the summer, "Tian" represents chrysanthemum in the autumn, "Hua" is on behalf of plum in the winter, which gives persons the overall sense of time, meaning good luck and happiness forever continues.

In addition, the nave painting "big vase" is also very representative. The bottle is inserted with flowers of the four seasons: peony, lotus, chrysanthemum, plum blossom, rich multicolored decorations, with luxuriant foliage, filled the entire screen, meaning bottle (safe) in the four seasons ,it is prosperous in the four seasons. On the surface, it is peace and safe in four seasons of all the year, it is actually the expression that people wish peace forever. "Wu $\mathrm{Zi}$ Deng Ke." the painting is also used to show flowers representing the four seasons, meaning to hope own family every generations has a bright future, wealthy knowledge, make one's ancestors illustrious from time and space. An enlarged longevity in the painting of "Bai Hua Xian Shou", there are seasonal flowers in the middle, meaning that life is always in full bloom as prosperous as flowers, youth is eternal. The "six Shun" painting is decorated with flowers in four seasons, which means forever smooth. 
The flowers are the most charming and the most beautiful images in the plants, it has become excellent material that artists created, it can not only give people the vision of the natural beauty, people's psychological and emotional beauty will be met at the maximum in time and space .

\section{B. Express the concept of space-time with animals}

Animals in Wuqiang New Year paintings have auspicious meaning, such as deer means "Paul", crane means "Royal", monkey means "Hou", fish means "rich" etc. In addition to these good meanings, and part of animals, people use it to express their understanding of time, it is the twelve zodiac. The twelve zodiac, is an important part of Chinese traditional culture, which is made up of animals from twelve in nature, namely rat, ox, tiger, rabbit, dragon, snake, horse, sheep, monkey, cock, dog, pig in order to record change of years, order of recording time is arranged for the $\mathrm{Zi}$ rat, Chou ox, Yin tiger, Mao rabbit, Chen dragon, Si snake, Wu horse, Wei sheep, Shen monkey, You chicken, Xu dog, Hai pig. The twelve zodiac sequence is in a certain order, ancient scholars explain the twelve zodiac and sort according to activity law of various animal in nature, such as "black Gou, chaos, rat, nearly midnight when the occasion out activities, the chaos state between heaven and earth bite out of the gap 'rat bite days open', so $\mathrm{Zi}$ is rat." And so on, all kinds of animal have the corresponding hours. The famous Neo Confucianism in Song dynasty, Zhu Xi, based on cultural interpretation of the twelve zodiac, created a "Twelve Chen poetry", the twelve animals and the lunar mansions of the Heaven are linked, write from the middle of the night to hear mouse active alarm, has written "guest to barking rush boil tea, no club sell pork", activities of the 12 animals in the whole day were written according to the time sequence. This poem is easy to understand, be popular, and widespread, making Chinese people have more special feelings about the twelve zodiac, until today to record the change of years with twelve zodiac replacement.

The calendar paintings in Wuqiang New Year paintings are decorated with the twelve zodiac, a set of practice and aesthetic. Calendar paintings refer to New Year paintings in which the contents of solar term tables or solar term, in order to facilitate farming activities, which is very important in the countryside, every house will hang it, and it will be changed each year. According to Liu Kezhuang's Poetry, the poet in Song Dynasty said: "This year no one sends the calendar, and watch the older draws on the wall", visibly, calendar paintings have been quite popular in Song dynasty. Early calendar paintings "nine nine no-cold figure", "all start anew", then the contents and forms of calendar paintings are continually innovated, the "twenty-four solar term table", "six new year", "three years earlier to know", "six order" etc. appeared. These paintings have twelve Chinese Zodiac images, the time and subjects matching with the twelve zodiac representing the change of time, which not only makes he paintings beautiful, and add more functions of calendar paintings, which implies the cycles of time and eternal movement of time.

In addition to the twelve zodiac, door painting "rooster" is also expression about time and space. Based on the experience of life, cock will crow just before dawn, "when cocks crow, it dawns" which means a new day begins. The cock has characteristics of darkness and ghosts, meet brightness, so put the chicken on the door and drive away evils. In case that people fear the darkness, they always look forward to set the time in the early morning when there are no ghosts, bright moments. So in the New Year paintings, rooster means eternal morning, a new start.

Based on the life experience, "spring cattle" represents a special visual symbol. "Buy spring cattle, buy spring cattle, and then see calendar head, all are here, and the twenty-four solar term is in the above." This is the song about "spring cattle", and from this, "spring cattle" plays an important role in people's life .The cattle in the painting means that the spring comes, and it is to define the time symbol of early spring. The key of the whole year is spring, hard cattle and people devote to working with passion in a new year, and New Year paintings are put in the whole year. In the bedroom, time is always fixed in the spring, The good wishes, such as bumper grains are harvest, resources are rich, family are healthy, are pinned in this season in the new year, which makes people have full of hope for a better life, firmly believe to pursue a happy life, enhance motivation.

\section{Express the concept of space-time with scenes of characters}

The artists of Wu Qiang New Year paintings express the concept of time and space with the creation of human figure, which is a unique form of expression of Wuqiang New Year paintings. The painting "The six sons struggle for head" is a typical representative through human figure to express the time concept. There are three reasons: Firstly, the figure that six sons struggle for head early and four sons struggle for the head only appeared in the "nine away cold figure", these graphs is mainly used to count nine cold days in winter, it is an ancient calendar painting; later works "six order" its screen is that six sons struggle for head, is the calendar painting. Secondly, in the painting of "The six sons struggle for head" there are the twelve zodiac, the twelve zodiac also appeared only in calendar painting, the twelve zodiac does not represent the twelve years, it is the expression of the cycle time. The shape of "the twelve zodiac" is round, six sons are alive, without beginning and end, move in circles, dynamic, it matches with just the concept of time recording twelve zodiac. Thirdly, the two sons share a head, and it matches with an hour, sixty minutes are an hour, six subs just form a "one", "one" is infinite, perfect. Later works of "six for head" painting not only is decorated with twelve zodiac, the four corners in the painting are decorated with flowers, representing the four seasons, the whole themes of the time are showed perfectly. "Four sons struggle for head" is an expression of the cycle of seasons.

There is the expression of time with the movement of positions in Wuqiang New Year paintings. "Spring cattle busy Immortal " is a very practical calendar painting in the early rural, named because of spring cattle and busy Immortal, its main function is to tell people the spring time to work, to guide the agricultural production, so the image of the cattle and busy Immortal are regarded as the main images. 
There is no calendar early in the countryside, farmers worked all activities of harvest and so on according to the lunar solar term as a primary reference standard, and the lunar solar term has no fixed date, the artists created "spring cattle busy Immortal " to inform farmers time to work. Such as "early spring" in the twelfth lunar month, busy Immortal stood in the front of cattle, meaning that spring comes early; such as "spring" in the lunar January, busy Immortal stands in back of cattle, meaning farming time will be late, it does not apply for early farming 1 .This expression method of time is very direct, easy to understand, and also very interesting.

Time and space in Wuqiang New Year paintings were shown with all directions. The space of all directions is to abandon the fixed azimuth, distance in the retina of monocular focusing junction, in the way of scattering or depth to acquire another space reality. The perception of space of China's ancient came from a spirit transcendence, which is "three views" of day, ground, people, or it is also the hierarchy of a section, the qualitative description. Such as Mawangdui non clothing painting unearthed in Hunan, the three levels of heaven, earth, are shown in a silk, won one world unity of panoramic panorama, which shows: a long time ago Chinese realized, to realized a complete world, there must be a spirit transcendence. This space transcendence thought in Wuqiang new year paintings still have the full performance. "All immortal of heaven and earth" includes longitudinal The Heaven Immortal, The Ground Immortal, The Human Immortal, ghosts, Confucianism, Buddhism, Taoism and other multi space of the folk deities, they were integrated into a painting, and each had its place, shared human fireworks. The uppermost in all the God is Buddhist "Buddha III Kasyapa Buddha": in the past,Buddha Sakyamuni, real Buddha was Sakyamuni, the future Buddha was Maitreya Buddha, a painting contained horizontally the past, the present, the future, and longitudinally, cross regional such as space, time and space range, it is amazing. Similarly, there are "three realms", "ten -party Zhenzai", according to each different firm's operating time length, the number of the fairy and layers of block are different, but all appear with the full range of time and space.

Another expression of time and space in Wuqiang New Year paintings is the transcendence of visual recognition of time and space that is all are perfect Negative observation method of fixed point of view, observe the objects from different viewpoints of time and space, and then put them on the same screen integration. Because different observation angles and combining ways are freely chosen, so the painting format has strong flexibility, thus a more subjective, free creating form is formed. Folk artists believe everything they see varies from minutes to minutes, this is their understanding and awareness about the movement of life, so in art creation, according to their own understanding of life form, re-process and combine, produce a new painting effect, and shows strong tension. Such as "ten men are busy ", "ten women are busy ", farmers are often busy", "textile painting", "scholar, farmer, artisan and merchant" and so on, are models which put different scenes of space and time in the same painting.
The festivals in each new year are the time points of the general public life, it regards the annual as the cycle, based on timing of the four seasons, depend on some natural characteristics and biological characteristics to calculate the changes of time and space. In the farming period, human timing must adapt to the natural timing, and summarize the time system in the process, guide the production through utilizing this system, and it's a very important part in people's life. After a long-term accumulation, the understanding of fixed time and space is formed in the heart of people, in the creation of New Year paintings, according to their own understanding of time and space, people consciously or unconsciously reveal. "Observe things and know climate", and achieve "legislation is to make meaning".

\section{CONCLUSION}

The unique expressing way of time, space Wu Qiang new year paintings is applied in the modern design ideas, it gets rid away from the original habitual thinking mode of time and space, it will give our designs in the enlightenment from a new perspective, new thinking ways, the plane breakthrough the concept of multi dimension of space and time, the our design and creative space is more enlarged. For modern designers, know better and master the multidimensional space, jump out of normal vision, breakthrough in $2 \mathrm{D}$ or $3 \mathrm{D}$ space limitations, seeking specification of space, it can enrich our modern design.

\section{REFERENCES}

[1] Sui Jie Wu Qiang New Year Paintings [M]. Beijing: Science Press .2009 Version 75

[2] Lv Shengzhong style origination· volumes · [M] Beijing: Peking University Press, 2009, p. 94

[3] Zhang Xike. Chinese twelve Zodiac Culture Exploration [J] Oriental Forum .2008 The Third Journal. 